\title{
Antibiotic resistance profile of Escherichia coli strains isolated in an infectious diseases clinic
}

\begin{tabular}{c}
\hline Lucian Giubelan, Iulian Diaconescu, Livia Dragonu, \\
Andreea Cristina Stoian, Florentina Dumitrescu \\
Infectious Diseases Clinic, University of Medicine and Pharmacy, Craiova, Romania \\
\hline
\end{tabular}

\begin{abstract}
Objective. To establish the resistance profile of Escherichia coli (EC) strains isolated in the Infectious Diseases Clinic from Craiova.

Material and methods. Retrospective study (January 2017-December 2018); EC strains were identified using the automated Vitek 2 system, which subsequently established their susceptibility to antimicrobials (usual testing for 17 antibiotics, extended testing for another 9); for each strain the Multiple Antibiotic Resistance Index (MAR) was calculated (limits: 0-1); the information was entered into an Excel database. 128 strains were tested in 2017 and another 592 in 2018. The percentage of MDR strains was calculated based on internationally accepted definitions.

Outcomes. 720 strains were identified, the vast majority isolated by urine culture (493 strains $-68.47 \%$ ). Demographic data: 508 strains (71\%) were isolated in adult subjects, $441(61 \%)$ in female patients, 411 $(57 \%)$ in patients living in urban areas. The overall value of MAR was 0.23 . Over $80 \%$ of EC strains were susceptible to Ertapenem, Meropenem, Amikacin, Polymyxin E, Gentamicin, Tobramycin, Cefoxitin; susceptibility between 60 and $79 \%$ were observed for Cefepime, Ceftriaxone, Ciprofloxacin, Ceftazidime, Levofloxacin, Imipenem, Nitrofurantoin, Cefazolin, and between 40 and 59\% for Trimethoprim-Sulfamethoxazole and Minocycline. Less than $39 \%$ of the strains were susceptible to Ampicillin ( \pm Sulbactam), Piperacillin ( \pm Tazobactam), Ticarcillin ( \pm Clavulanate), Aztreonam, Pefloxacin. Resistance to the main classes of antibiotics is consistent with European and national data, except for a much higher percentage of carbapenemresistant strains. Over $50 \%$ of isolates are multidrug-resistant; $48.3 \%$ of the strains come from sources where there is significant contact with antimicrobials.

Conclusions. Over $80 \%$ of the isolated strains are susceptible to Ertapenem, Meropenem, Amikacin, Polymyxin E, Gentamicin, Tobramycin, Cefoxitin; more than $4.5 \%$ of the strains demonstrate resistance to the carbapenem class; over $50 \%$ of isolates are multidrug-resistant; $48.3 \%$ of the strains come from sources where there is significant contact with antimicrobials.
\end{abstract}

Keywords: Escherichia coli, resistance to antimicrobials, MAR

\section{BACKGROUND}

Escherichia coli (EC) is a Gram-negative bacteria (GNB) of great importance in human pathology, generating a wide range of infections (mainly urinary and gastrointestinal, but also systemic, meningeal or lung infections). From an epidemiological point of view, the bacteria is able to generate large-scale epidemics, such as the one in 2011 (almost 4,000 cases and 53 deaths) [1-3].
Antimicrobial resistance is a major public health problem worldwide, recognized as such by the World Health Organization (WHO), which in September 2016 convened a General Assembly dedicated entirely to this topic, on which occasion it was launched a Global Plan of Action Against Antimicrobial Resistance [4]. In 2017 WHO published a list of 12 bacteria for which development of new antibiotics is urgently needed; among these, priority 1 (critical) includes 
Enterobacteriaceae (which includes EC) producing broad-spectrum beta-lactams and carbapenems [5].

The European Center for Disease Prevention and Control (ECDC) periodically publishes reports on antibiotic resistance for the main "problematic" bacteria; the last report was published in 2019 and analyzes the situation by the end of the previous year [6].

At the national level, the CARMIN-ROM project has so far published 6 reports (the last in 2019, analyzing the available data until the end of 2017) in which it analyzes aspects of antibiotic resistance [7].

It is important for the clinicians to know the antibiotic resistance pattern of EC strains in the area in which they operate, for a rapid and appropriate therapeutic response

\section{OBJECTIVE}

To establish the resistance profile of Escherichia coli (EC) strains isolated in the Infectious Diseases Clinic from Craiova.

\section{METHOD}

Retrospective study (January 2017-December 2018) based on data from the hospital laboratory records; GNB and, particularly, EC strains were identified using the automated Vitek 2 system, which subsequently established their susceptibility to antimicrobials (usual testing for 17 antibiotics $97.08 \%$, extended testing for another $8-1.38 \%$ of EC, 10 bacterial strains $-1.38 \%$ were tested to 16 antimicrobials, while one isolate were tested to only 14 antibiotics); for each strain the Multiple Antibiotic Resistance Index (MAR) was calculated (limits: 0-1); the information was entered into an Excel database. 128 strains were tested in 2017 and another 592 in 2018.

\section{RESULTS}

720 strains were identified (out of a total of 1358 GNB strains, EC being the most important bacterium in this group $-53.01 \%$ ).

Demographic data: the median age of the patients was 53 years (limits: $<1$ year -94 years); 508 strains (71\%) were isolated in adult subjects, $441(61 \%)$ in female patients, $411(57 \%)$ in patients living in urban areas.

The vast majority of ECs were isolated by urine culture (493 strains - 68.47\%). An exact situation of the pathological products from which the bacteria was isolated is presented in Table 1. It should be noted that, in pediatric patients, the EC was only isolated from urine or faecal samples. No adult patients had positive EC stool culture.

TABLE 1. Type and number of samples from which the EC was isolated

\begin{tabular}{|l|c|}
\hline Pathological sample & No. of isolates \\
\hline Urine culture & 493 \\
\hline Stool culture & 113 \\
\hline Sputum & 79 \\
\hline Blood culture & 22 \\
\hline Wound culture & 4 \\
\hline Bronchial aspirate & 3 \\
\hline Vaginal discharge & 3 \\
\hline Pericar & 1 \\
\hline Cannula secre & 1 \\
\hline Urinary probe culture & 1 \\
\hline
\end{tabular}

The EC resistance profile for a specific antibiotic is shown in Table 2.

Over $80 \%$ of EC strains were susceptible to Ertapenem, Meropenem, Amikacin, Polymyxin E, Gentamicin, Tobramycin, Cefoxitin; Susceptibilities between 60 and 79\% were observed for Cefepime, Ceftriaxone, Ciprofloxacin, Ceftazidime, Levofloxacin, Imipenem-cilastatin, Nitrofurantoin, Cefazolin, and between 40 and 59\% for Trimethoprim-Sulfamethoxazole and Minocycline. Less than 39\% of the strains are susceptible to Ampicillin ( \pm Sulbactam), Piperacillin ( \pm Tazobactam), Ticarcillin ( \pm Clavulanic acid), Aztreonam, Pefloxacin. Figure 1 graphically shows the susceptibility pattern of EC strains.

The overall value of MAR was 0.23 (compared to 0.32 for all strains of isolated Gram-negative germs unpublished data). Figure 2 graphically shows the distribution of EC strains according to the values of MAR fractions.

Table 3 shows the variation of the percentage of EC strains resistant to different classes of antibiotics in two consecutive years (2017 and 2018).

\section{DISCUSSIONS}

In the European Union (EU) resistance to aminopenicillin in 2018 was high (on average 57.4\%), for Romania being above this value $(62.2 \%$, 8th place in the EU), but decreasing compared to 2017 (68.2\%), a percentage also confirmed by the CARMIN-ROM 2017 study (68.7\%) [6,7]. In the Infectious Diseases 
TABLE 2. Resistance profile of EC strains according to the tested antibiotic

\begin{tabular}{|l|c|c|c|c|c|c|}
\hline \multicolumn{1}{|c|}{ Antimicrobial } & s & i & r & s\% & i\% & r\% \\
\hline Amikacin & 681 & 27 & 11 & 94.7 & 3.8 & 1.5 \\
\hline Ampicillin & 220 & 19 & 474 & 30.9 & 2.7 & 66.5 \\
\hline Ampicillin-Sulbactam & 296 & 99 & 317 & 41.6 & 13.9 & 44.5 \\
\hline Aztreonam* & 6 & 3 & 8 & 35.3 & 17.6 & 47.1 \\
\hline Cefazolin & 450 & 0 & 262 & 63.2 & 0.0 & 36.8 \\
\hline Cefepime & 567 & 64 & 82 & 79.5 & 9.0 & 11.5 \\
\hline Cefo & 612 & 21 & 80 & 85.8 & 2.9 & 11.2 \\
\hline Ce azidime & 554 & 102 & 64 & 76.9 & 14.2 & 8.9 \\
\hline Ce xoß7e & 557 & 31 & 124 & 78.2 & 4.4 & 17.4 \\
\hline Cipr xacin & 557 & 5 & 156 & 77.6 & 0.7 & 21.7 \\
\hline Ertapenem & 689 & 0 & 24 & 96.6 & 0.0 & 3.4 \\
\hline Gentamicin & 640 & 5 & 75 & 88.9 & 0.7 & 10.4 \\
\hline Imipenem-cilasta & 13 & 0 & 4 & 76.5 & 0.0 & 23.5 \\
\hline Lev xacin & 549 & 4 & 163 & 76.7 & 0.6 & 22.8 \\
\hline Meropenem & 688 & 6 & 25 & 95.7 & 0.8 & 3.5 \\
\hline Minocycline* & 8 & 4 & 4 & 50.0 & 25.0 & 25.0 \\
\hline Nitrofurantoin & 521 & 152 & 37 & 73.4 & 21.4 & 5.2 \\
\hline Pe xacin* & 5 & 0 & 10 & 33.3 & 0.0 & 66.7 \\
\hline Piperacillin & 237 & 8 & 474 & 33.0 & 1.1 & 65.9 \\
\hline Piperacillin-Tazobactam* & 6 & 5 & 6 & 35.3 & 29.4 & 35.3 \\
\hline Polymixyn E* & 16 & 0 & 2 & 88.9 & 0.0 & 11.1 \\
\hline Ticarcillin* & 3 & 0 & 14 & 17.6 & 0.0 & 82.4 \\
\hline Ticarcillin-Clavulanate* & 1 & 19 & 0 & 5.0 & 95.0 & 0.0 \\
\hline Tobramycin & 629 & 4 & 87 & 87.4 & 0.6 & 12.1 \\
\hline Trimethoprim- & 442 & 0 & 275 & 61.6 & 0.0 & 38.4 \\
\hline Sulphamethoxazole & & & & & & \\
\hline
\end{tabular}

Legend: $s=$ susceptible, $i=$ dose-dependent susceptibility, $r=$ resistant; ${ }^{*}=$ extended testing

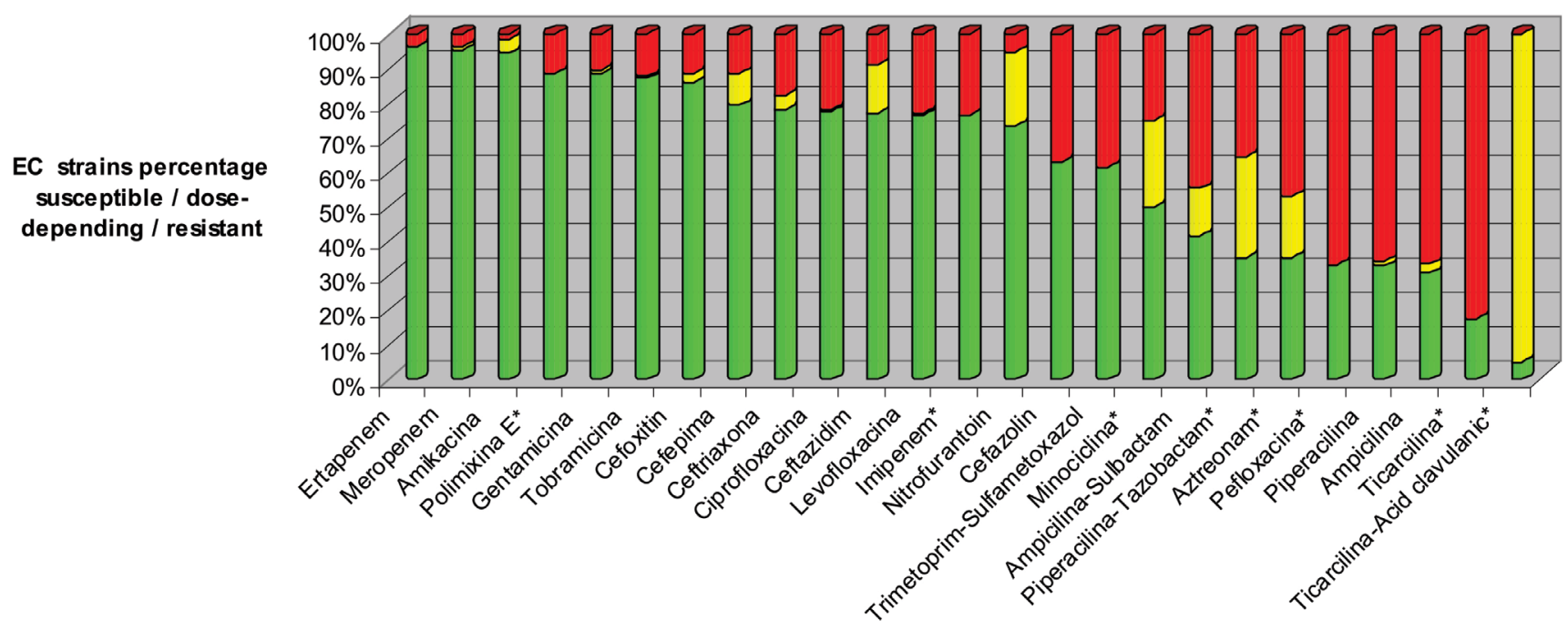

\section{पS पI पR}

FIGURE 1. Antibiotic susceptibility profile of isolated EC strains. Legend: $S=$ susceptible (green) $I=$ dose-depending (yellow), $R$ = resistant strain (red)

Clinic from Craiova, the percentage of aminopenicillin resistance was $70.31 \%$ in 2017 (90 resistant strains out of a total of 128 tested for Ampicillin and Ampicillin/Sulbactam), respectively $64.86 \%$ in 2018 (384 
MAR Escherichia coli

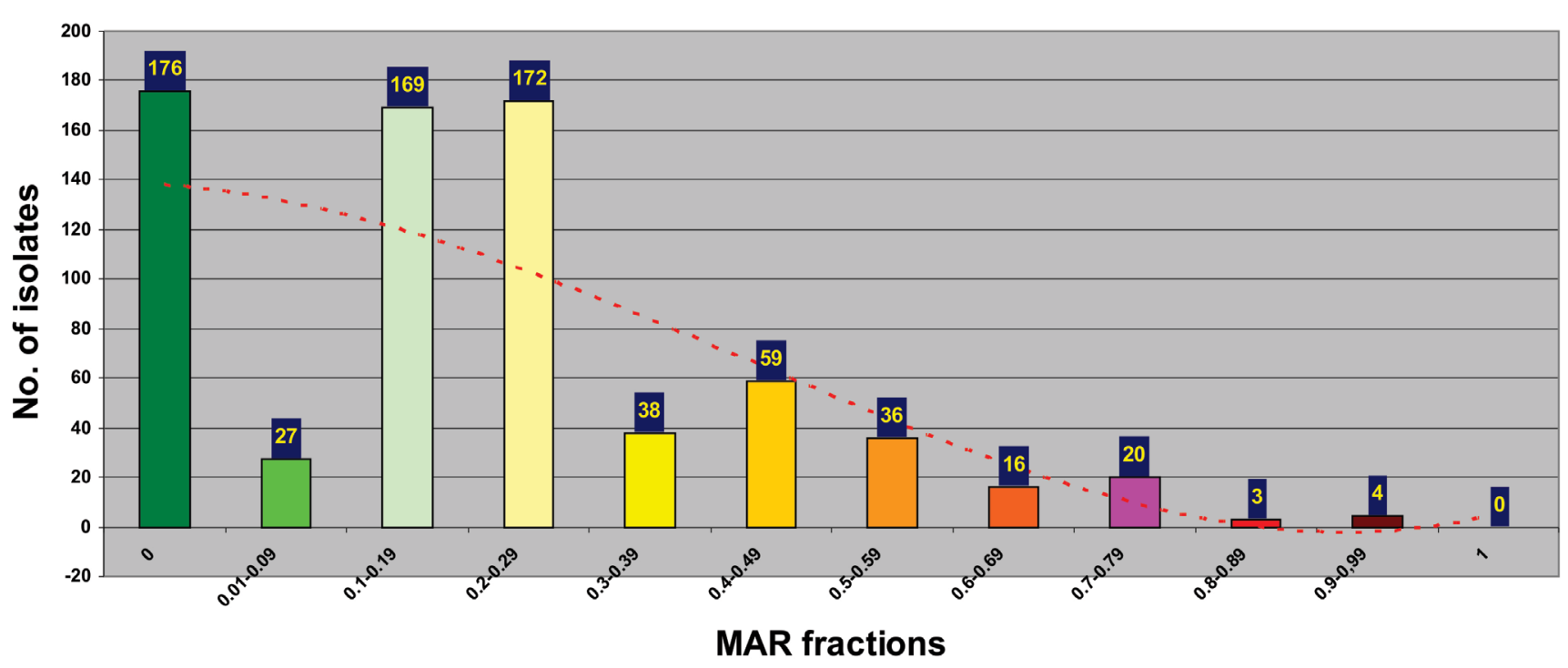

FIGURE 2. Distribution of EC strains according to MAR fraction values

TABLE 3. Percentage of strains resistant to different classes of antimicrobials, 2017 vs. 2018

\begin{tabular}{|c|c|c|c|c|c|}
\hline & AminoP & CEF 3 & FQ & AG & CarbaP \\
\hline & \multicolumn{5}{|l|}{2017} \\
\hline total EC & 128 & 128 & 128 & 128 & 128 \\
\hline $\mathbf{r}$ & 90 & 32 & 30 & 11 & 6 \\
\hline$\%$ & 70.31 & 25.00 & 23.44 & 8.59 & 4.69 \\
\hline \multicolumn{5}{|l|}{2018} \\
\hline total EC & 592 & 592 & 592 & 592 & 592 \\
\hline $\mathbf{r}$ & 384 & 100 & 134 & 82 & 27 \\
\hline$\%$ & 64.86 & 16.89 & 22.64 & 13.85 & 4.56 \\
\hline
\end{tabular}

Legend: AminoP = aminopenicillins, CEF $3=3$ rd generation cephalosporins,

$F Q=$ fluoroquinolones, $A G=$ aminoglycosides, $C a r b a P=$ carbapenems,$E C=$ Escherichia coli,

$r=$ (no. of strains) resistant (to antimicrobials)

resistant strains out of 592 tested), values that overlap with those previously presented. It should be noted that the addition of the beta-lactamase inhibitor (Sulbactam) did not influence the resistance profile, all strains tested being either sensitive or resistant to both antimicrobials.

Resistance to 3rd generation cephalosporins was on averaged $15.1 \%$ in the EU (2018), while Romania ranked 7 th, with an average value of $20.2 \%$ (slightly lower than in $2017-22 \%$ ); according to the CARMIN-ROM study, in 2017 resistance to this class was $20.1 \%$ [6,7]. The data obtained from this study reveal an average level of resistance of $25 \%$ in 2017 (32 strains tested, resistant to Ceftriaxone, Ceftazidime or both, out of a total of 128 strains tested) and $16.84 \%$ in 2018 (100 resistant strains out of a total of 592). This decrease is unlikely to be the effect of antimicro- bial use policies, but rather reflects an improvement in the situation as a result of a larger number of tests performed.

Fluoroquinolone resistance had an average value of $25.3 \%$ for the EU as a whole, Romania registering a percentage above it (11th place), increasing from $26.4 \%$ (2017) to $29.1 \%$ (2018); the percentage in 2017 is also confirmed by the CARMIN-ROM study (28.4\%) [6,7]. Our data are below these values (23.42\% in 2017 - 30 strains resistant to Ciprofloxacin, Levofloxacin or both, out of a total of 128 strains tested, respectively $22.63 \%$ in 2018,134 resistant bacteria out of 592 tested).

Regarding the resistance to aminoglycosides, the average European level was $15.9 \%$, Romania occupying the 10th place with a percentage value of 15.2 in 2017, decreasing to 12.8 for 2018 ; the percentage in 
2017 is also confirmed by the Romanian national multicenter study (15.9\%) [6,7]. The data obtained by us show a percentage value of $8.59 \%$ in 2017 (11 strains resistant to Amikacin, Gentamicin, Tobramycin or combinations thereof, out of a total of 128 strains tested), increasing to $13.85 \%$ in 2018 (82 resistant strains out of 592 tested).

For 2018, the percentage of EC resistance to carbapenems in the EU is subunit, except for Greece, Bulgaria and Cyprus; The CARMIN-ROM study identified only two resistant strains out of 511 tested $[6,7]$. Our data show a different and worrying situation, the percentage of resistance to Meropenem, Ertapenem or both being 4.62 in 2017, respectively 4.56 in 2018. Due to the small number of strains tested we did not take into account the data for Imipenem-cilastatin .

Polymyxin-resistant strains have been described worldwide [8-10], this class of antibiotics being considered a last line of defense against GNB [11]. Two colistin-resistant strains were identified in our clinic,

\section{REFERENCES}

1. Frank C, Werber D, Cramer JP et al. Epidemic profile of Shiga-toxinproducing Escherichia coli 0104:H4 outbreak in Germany. N Engl J Med. 2011;365(19):1771-1780.

2. Altman $M$, Wadl M, Altman $D$ et al. Timelines of surveillance during outbreak of Shiga toxin-producing Escherichia coli, Germany, 2001. Emerg Infect Dis. 2011;17(10):1906-1909.

3. Buchholz U, Bernard H, Werber D et al. German outbreak of Escherichia coli 0104:H4 associated with sprouts. N Engl J Med. 2011;365:1763-1770.

4. WHO. 2015 Global action plan on antimicrobial resistance, available at: https://www.who.int/antimicrobial-resistance/publications/ global-action-plan/en/.

5. WHO. $2017 \mathrm{WHO}$ publishes list of bacteria for which new antibiotics are urgently needed, available at: https://www.who.int/news-room/ detail/27-02-2017-who-publishes-list-of-bacteria-for-which-new-antibiotics-are-urgently-needed.

6. ECDC. 2019 Surveillance of antimicrobial resistance in Europe 2018, available at: https://www.ecdc.europa.eu/sites/default/files/ documents/surveillance-antimicrobial-resistance-Europe-2018.pdf.

7. Popescu GA, Şerban R, Niculcea A. 2019 CARMIN-ROM 2017 (Consumul de antibiotice, rezistenţa microbiană şi infecţiile asociate asistenţei medicale (nosocomiale) în România - 2017), 41-42, available at: https://www.cnscbt.ro/index.php/analiza-date-suprave- out of a total of 16 tests. However, both strains have shown susceptibility to other antimicrobials.

According to the definition criteria for multidrug resistance (MDR) and extended drug-resistance (XDR) [12] we have identified $51.94 \%$ of EC strains as MDR, but we did not register any XDR strains.

By similarity with the criteria defined by Krumperman [13], we have identified 348 EC strains $(48.33 \%)$ with a MAR index of over 0.2 . This suggests that almost half of the analyzed strains come from sources with significant exposure to antibiotics (hospital-related).

\section{CONCLUSIONS}

Over $80 \%$ of the isolated strains are susceptible to Ertapenem, Meropenem, Amikacin, Polymyxin E, Gentamicin, Tobramycin, Cefoxitin; more than $4.5 \%$ of the strains demonstrate resistance to the carbapenem class; over $50 \%$ of isolates are multidrug-resistant; $48.3 \%$ of the strains come from sources where there is significant contact with antimicrobials.

Conflict of interest: none declared Financial support: none declared

ghere/infectii-nosocomiale-1/1309-consumul-de-antibiotice-rezistenta-microbiana-si-infectii-asociate-asistentei-medicale-nosocomiale-in-romania-2017/file.

8. Yin W, Li H, Shen $\mathrm{Y}$ et al. Novel plasmid-mediated Colistin resistance gene mcr-3 in Escherichia coli. mBio. 2017;8(3):e00543-17.

9. Mediavilla JR, Patrawalla A, Chen L et al. Colistin- and Carbapenemresistant Escherichia coli harboring mcr-1 and blaNDM-5, causing a complicated urinary tract infection in a patient from United States. mBio. 2016;7(4):e001191-16.

10. Carattoli A, Villa L, Feudi $C$ et al. Novel plasmid-mediated Colistin resistance mcr-4 gene in Salmonella and Escherichia coli, Italy 2013, Spain and Belgium, 2015 to 2016. Euro Surveill. 2017;22(31):30589.

11. El-Sayed Ahmed MAEG, Zhang LL, Shen C et al. Colistin and its role in the Era of antibiotic resistance: An extended review (2000-2019). Emerg Microbes Infect. 2020;9(1):868-885.

12. Magiorakos AP, Srinivasan A, Carey RB et al. Multidrug-resistant, extensively drug-resistant and pandrug-resistant bacteria: an international expert proposal for interim standard definitions for acquired resistance. Clin Microbiol Infect. 2012; 18:268-281.

13. Krumperman PH. Multiple antibiotic resistance indexing of Escherichia coli to identify high-risk sources of fecal contamination of foods. Appl Environ Microbiol. 1983;46(1):165-170. 